\title{
Implementation and Early Results of Extended Lymph Node Dissection for Gastric Cancer in a Non-Specialized Western Center ${ }^{\S}$
}

\author{
Marco Catarci ${ }^{*}, 1,3$, Sabrina Ghinassi ${ }^{1}$, Antonio Di Cintio ${ }^{1}$, Leonardo Antonio Montemurro ${ }^{1}$, \\ Luigi Pinnarelli ${ }^{2,3}$, Leonardo Leone ${ }^{1}$, Graziano Longo ${ }^{1}$ and Giovanni Battista Grassi ${ }^{1}$ \\ ${ }^{I}$ Departments of Surgery, ${ }^{2}$ Clinical Epidemiology, ${ }^{3}$ Center for Clinical Evidence, San Filippo Neri Hospital, Rome, Italy
}

\begin{abstract}
Background: The optimal degree of lymph node dissection for gastric cancer is still matter of debate. Particularly, there are serious doubts about the reproducibility of extended lymph node dissection in western surgical units, and no studies to date have investigated factors influencing early results (mortality, major morbidity and reoperation rates) during the learning curve.

Methods: Univariate and multivariate analysis of 19 variables on a prospective series of 313 consecutive resections for gastric cancer performed by ten different surgeons. Endpoints were mortality, major morbidity and reoperation rates, calculated within 60 days form the operation.

Results: Early results were all independently influenced by the presence of comorbidities alone. ASA status III-IV vs I-II determined a higher operative mortality rate $(11.9 \%$ vs $0.5 \%$; Odds Ratio 12.3 ; $95 \%$ c.i. 1.53 to 98.1 ; p .018), a higher major morbidity rate $(39.7 \%$ vs $16.6 \%$; Odds Ratio $2.71 ; 95 \%$ c.i. 1.51 to $4.88 ; \mathrm{p} .0008)$ and a higher reoperation rate (9.5\% vs 2.1\%; Odds Ratio 4.81; $95 \%$ c.i. 1.51 to 15.3 ; p .008).

Conclusions: Extended lymph node dissection can be safely implemented into the clinical practice of a non-dedicated western institution by providing adequate coaching from more expert surgeons. This implementation protocol led to acceptable rates of operative morbi-mortality, independently influenced only by the comorbidity status.
\end{abstract}

Keywords: Stomach neoplasms, Surgery, lymph node dissection.

\section{INTRODUCTION}

The optimal degree of lymph node dissection for gastric cancer is still matter of debate. Extended lymph node dissection routinely performed in Eastern centers showed overall survival rates exceeding $70 \%$ at five years [1] with less than $1 \%$ operative mortality rates [2]. Several dedicated or specialized western centers have reproduced these results [3-8]. On the other hand, two large randomized trials in Europe $[9,10]$, have failed to show any survival advantage and unacceptable morbidity and mortality rates for extended lymph node dissection, mainly due to absence of proper training [11], questioning its widespread adoption in western clinical practice. The purpose of this study is a prospective evaluation of factors influencing early results during implementation of extended lymph node dissection in our surgical unit.

\section{MATERIALS AND METHODS}

From January 1998 to September 2008, 422 consecutive patients were submitted to surgery for gastric adenocarcinoma in our unit. Of these, 69 patients $(16.4 \%)$

\footnotetext{
*Address correspondence to this author at the UOC Chirurgia Generale \& Oncologica, Dipartimento di Chirurgia, ACO San Filippo Neri, Via G. Martinotti, 20 - 00135 Roma, Italy; Tel: +39 329 8610040; Fax: +39 06 33062232; E-mail: m.catarci@sanfilipponeri.roma.it

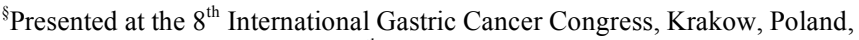
June $10-13,2009$ and at the $111^{\text {th }}$ Congress of Società Italiana di Chirurgia, Rimini, Italy, October 23-25, 2009.
}

were not resected (23 staging laparotomy/laparoscopy alone, 46 gastro-jejunal bypass or feeding jejunostomy). The remaining 353 cases $(83.6 \%)$ were submitted to surgical resection of the tumor.

Forty cases were excluded from this study: 22 cases in which the neoplasm was located in the gastric stump after previous gastric resection; 8 cases in which poor general conditions and emergency (bleeding) or a synchronous malignant neoplasm in other organs prompted a palliative resection with $\mathrm{D}_{0}$ lymph node dissection; 6 cases submitted to resection of a recurrent tumor and 4 cases submitted to neo-adjuvant chemotherapy. The remaining 313 cases constitute the population of this study. All perioperative data of these patients were prospectively recorded into a database, and outpatient follow-up scheduled every six months during the first three years and yearly thereafter, up to the study censor scheduled at November $30^{\text {th }}, 2008$.

\section{Setting}

All the operations were performed according to a standardized protocol of pre-, intra- and post-operative care in a tertiary care, 700-beds hospital of the Italian National Health System. In 1998, extended lymph node dissection $\left(\mathrm{D}_{2}\right)$ according to the JGCA [12] was introduced into the clinical practice of the unit. Two surgeons (MC, GBG) with previous specific training in extended lymph node dissection obtained through stages in Japanese surgical centers, personally monitored its implementation and diffusion, coaching the other eight surgeons participating in the study. 


\section{Patient-Related Variables}

There were 178 males and 135 females; mean \pm SD age was $67.3 \pm 11.6$ years (median 69; range 32-95) and it was categorized according to its median value. Concerning the presence of comorbidities, patients were classified utilizing the American Society of Anesthesiologists system: 187 were ASA class I-II and 126 ASA class III-IV. Body mass index (BMI), expressed as $\mathrm{Kg} / \mathrm{m}^{2}$, mean $\pm \mathrm{SD}$ was $25.4 \pm 3.7$ (median 25.4; range 15.2-37.9); for analytic purposes, it was categorized according to its median value.

\section{Neoplasm-Related Variables}

Location of the tumor was classified according to JGCA rules [12] into lower third, middle third and upper third. Eight cases of diffuse tumors (Borrmann IV type) were classified according to their prevalent location and 15 cardia cancers (Siewert [13] type II) were classified as upper third tumors.

The mean \pm SD maximum diameter of the tumor was $47.2 \pm 27.8 \mathrm{~mm}$ (median 40; range 5-160) and it was classified according to its median value. The UICC-TNM staging system, Fifth Edition [14], was used to define depth of invasion ( $\mathrm{pT})$, nodal status $(\mathrm{pN})$, distant metastases $(\mathrm{pM})$, stage grouping and grading. Microscopic type was defined according to Lauren [15].

\section{Treatment-Related Variables}

All operations were carried out in an elective setting under a strict perioperative care protocol including antibiotic and antithromboembolic prophylaxis. Access was always through a laparotomic approach, either a midline or a bilateral subcostal incision, depending upon body shape, tumor location and surgeon's preferences. Peritoneal lavage for cytopathologic examination was always obtained in any case with serosal tumor or beyond. A distal subtotal gastrectomy $(\geq 2 / 3)$ was carried out whenever it was possible to obtain a $4 \mathrm{~cm}$ macroscopic proximal margin from the edge of the tumor. In closer proximity of this margin and in case of any doubt, a frozen section of the resection margin was obtained and a total gastrectomy eventually performed. In all other cases, a total gastrectomy, eventually extended to lower esophagus through a trans-hiatal approach, was performed. Only in four upper third locations a proximal subtotal gastrectomy was carried out, and in two of these a right thoracotomy was added to control the upwards esophageal spread of the neoplasm. Digestive tract continuity was always restored through a Roux-en-Y manual gastro-jejunal or stapled esophago-jejunal anastomosis.

A standard $\mathrm{D}_{2}$ (extended) lymph node dissection according to the JGCA [12] was performed in 190 cases. In 27 of these, when the tumor was located in the lower third and was macroscopically infiltrating the serosal layer or beyond $\left(T_{3}-T_{4}\right)$, dissection of lymph nodes posterior to the common hepatic artery and of the hepatic pedicle was added. A more limited lymph node dissection was performed in the remaining 123 cases. $\mathrm{A}_{1_{\alpha}}$ lymph node dissection, namely removal of the first tier of perigastric nodes and of left gastric artery nodes, was performed in 68 patients with higher operative risk (ASA class III-IV); a $\mathrm{D}_{1 \beta}$ lymph node dissection, namely removal of the first tier of perigastric nodes, of left gastric artery, anterior to the common hepatic artery and celiac artery nodes, was performed in 55 patients with tumor located in the middle or upper third on the lesser curve and/or on the anterior wall.

Adjacent organs were removed en-bloc only when macroscopically invaded by the tumor. A pancreaspreserving splenectomy [16], was performed with a total gastrectomy in case of upper third locations along the greater curvature and/or on the posterior wall. A prophylactic cholecystectomy was performed in all $\mathrm{D}_{2}$ resections. At the study censor, seven out of ten participating surgeons performed at least ten cases. One surgeon (GBG, surgeon \#1) performed more than $50 \%$ of the operations. All the surgical specimens were formalin-fixed, sent en-bloc and processed as usual by ten pathologists who also performed lymph node retrieval without any fat-clearing method. The mean \pm SD number of examined lymph nodes per patient was $28.3 \pm 14.1$ (median 26, range $2-78$ ); It was $17.8 \pm 9.4$ (median 15, range 2-52) after $\mathrm{D}_{1 \alpha-\beta}$ lymph node dissection and $34.0 \pm 13.9$ (median 32, range 9-78) after $\mathrm{D}_{2}$ lymph node dissection. Surgical radicality $(\mathrm{pR})$ was defined according to UICC-TNM staging system [14]. Time-trend performance was evaluated including the accrual year as an interval variable.

\section{Endpoints and Statistical Analysis}

Early results (operative mortality, major operative morbidity and reoperation rates) were defined and calculated as any death, major complication or reoperation occurring within 60 days from surgery or during hospitalization when beyond the 60 days limit.

Major complications were defined and recorded as any adverse event requiring any kind of medical intervention and/or prompting any deviation from the perioperative protocol (duration of antibiotic or anticoagulant prophylaxis/therapy, blood transfusions, time to surgical drains removal, interventional radiology, reoperation, length of postoperative hospital stay, etc.), and classified as it follows: Anastomotic dehiscence, as any clinical or radiological evidence of esophago-jejunal or gastro-jejunal anastomotic leakage; Duodenal stump dehiscence, as any clinical or radiologic evidence of duodenal stump leakage; Abdominal haemorrhage, as any postoperative drop of haemoglobin level $>25 \%$ requiring blood transfusions; Abdominal abscess, as any clinical or radiological evidence of infected peritoneal fluid collection; Pancreatic fistula, as prolonged ( $>5$ days) drainage of fluid ( $>50 \mathrm{~mL}$ per day) with at least $3: 1$ concentration in amylase/lipase levels compared to serum; Major cardiac events, including myocardial ischemia/infarction, worsening of chronic heart failure or acute heart failure, new onset of dysrrythmias; Bronchopulmonar, including any infection, deep venous thrombosis and pulmonary embolism.

All data were analyzed with StatsDirect ${ }^{\mathcal{O}}$ statistical software, version 2.7.7 (StatsDirect Ltd., UK). Univariate analysis were performed applying Pearson correlation, Chi Square test or Fisher's exact test as appropriate. All significant variables were considered into a multivariate analysis using multiple linear or logistic regression as appropriate, with a non-conditional model [17]. The logistic model's goodness of fit was tested applying the likelihood 
ratio test and the Hosmer-Lemeshows test. Statistical significance was assumed for $\mathrm{p}$ values $<.05$.

\section{RESULTS}

At the study censor we recorded 16 deaths within 60 days from the operation (overall operative mortality rate $5.1 \%$ ), 81 major morbidities (overall major morbidity rate $25.9 \%$ ) and 16 reoperations (overall reoperation rate 5.1\%). Details about these results are described in Table $\mathbf{1}$.

Table 1. Details of Early Results

\begin{tabular}{|l|c|c|c|c|c|c|}
\hline \multirow{2}{*}{} & \multicolumn{2}{|c|}{ Deaths } & \multicolumn{2}{c|}{ Morbidities } & \multicolumn{2}{c|}{ Reoperations } \\
\cline { 2 - 8 } & No. & $\mathbf{\%}$ & No. & $\mathbf{\%}$ & No. & $\mathbf{\%}$ \\
\hline \hline Anastomotic dehiscence & 5 & 1.6 & 9 & 2.9 & 3 & 0.9 \\
\hline Duodenal stump dehiscence & 2 & 0.6 & 13 & 4.1 & 3 & 0.9 \\
\hline Abdominal haemorrhage & 2 & 0.6 & 9 & 2.9 & 4 & 1.3 \\
\hline Abdominal abscess & 2 & 0.6 & 18 & 5.9 & 6 & 2.0 \\
\hline Pancreatic fistula & -- & -- & 5 & 1.6 & -- & -- \\
\hline Major cardiac events & 5 & 1.6 & 12 & 3.8 & -- & -- \\
\hline Bronchopulmonar & -- & -- & 15 & 4.8 & -- & -- \\
\hline \multicolumn{1}{|c|}{ Total } & $\mathbf{1 6}$ & $\mathbf{5 . 1}$ & $\mathbf{8 1}$ & $\mathbf{2 5 . 9}$ & $\mathbf{1 6}$ & $\mathbf{5 . 1}$ \\
\hline
\end{tabular}

After univariate analysis (Tables 2-4), Gender, Age, ASA status and Type of lymph node dissection were considered into a multivariate analysis for operative mortality (Table 5); Age, ASA status, pM, Surgeon \#1 vs others and pR for operative morbidity (Table 6); ASA status was the only variable independently influencing both mortality and major morbidity rates. Concerning the reoperation rate, at univariate analysis it was significantly influenced only by ASA status (ASA III-IV vs I-II 9.5\% vs 2.1\%; Odds Ratio $4.81 ; 95 \%$ c.i. 1.51 to 15.3 ; p .008).

\section{DISCUSSION}

We failed to find any previous report analyzing factors influencing early results of extended lymph node dissection during the learning curve of ten different surgeons in a western non-dedicated surgical oncology unit. Overall rates of mortality (5.1\%), major morbidity (26.9\%) and reoperation $(5.1 \%)$ in this series are well within the range reported in western centers [6-8]. The nearest comparable study is probably the one from the European Oncology Institute [6], in which two experienced surgeons operated 250 consecutive patients from 1994 to 2002, with a lymph node dissection phylosophy very close to the one applied in the present series (splenectomy or splenopancreatectomy rate below 10\%). Mortality, morbidity and reoperation rates were $1.2 \%, 18 \%$ and $3.6 \%$, respectively. An Italian multicenter prospective trial [7], showed $0.6 \%$ mortality and $13.6 \%$ morbidity rates, but its exclusion criteria make it unsuitable for extrapolation into clinical practice. Looking at population-based studies, data from about 13,000 cases of the National Inpatient Sample [18], in the USA during the period 1998-2003 showed an operative mortality rate at $6 \%$; this rate was independently influenced by gender (higher in males), by the type of gastric resection (higher for total gastrectomy), by age (higher in older than 50 years) and by hospital caseload (higher in centers performing $<4$ cases per year). Similarly as in other western series [6-8], [18-21], early results in our experience were independently influenced only by the presence of significant comorbidities and no other variable.

Particularly, early results were not independently influenced by BMI in our series, in which more than half of the patients were overweight $(\mathrm{BMI}>25)$, confirming similar results in other western series [22,23]. Conversely, overweight significantly influenced morbidity and mortality rates in eastern series [24,25], in which only $10-15 \%$ of patients are overweight. This can reflect the fact that western surgeons are more used in operating on more "fatty" patients.

As expected, our analysis does not confirm the unacceptable morbidity (43-46\%) and mortality (10-13\%) rates in the $D_{2}$ arm of Dutch and British randomized trials $[9,10]$. This is probably due to the voluntary selection bias of patients in the $\mathrm{D}_{2}$ arm during this implementation study. The concept of standardizing extended lymph node dissection

Table 2. Early Results According to Patient-Related Variables

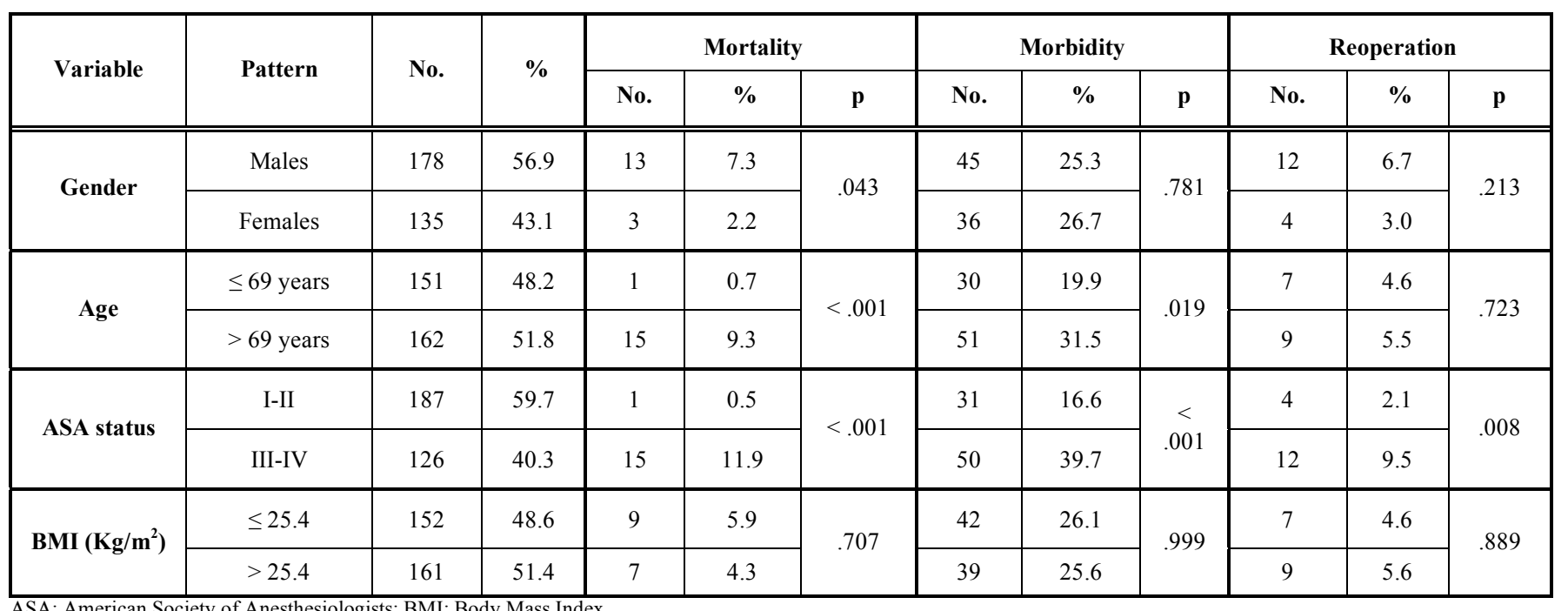


Table 3. Early Results According to Tumor-Related Variables

\begin{tabular}{|c|c|c|c|c|c|c|c|c|c|c|c|c|}
\hline \multirow{2}{*}{ Variable } & \multirow{2}{*}{\multicolumn{2}{|c|}{ Pattern No. }} & \multirow{2}{*}{$\%$} & \multicolumn{3}{|c|}{ Mortality } & \multicolumn{3}{|c|}{ Morbidity } & \multicolumn{3}{|c|}{ Reoperation } \\
\hline & & & & No. & $\%$ & $\mathbf{p}$ & No. & $\%$ & $\mathbf{p}$ & No. & $\%$ & $\mathbf{p}$ \\
\hline \multirow{3}{*}{ Location } & Lower $3^{\text {rd }}$ & 171 & 54.6 & 8 & 4.7 & \multirow{3}{*}{.718} & 46 & 26.9 & \multirow{3}{*}{.898} & 10 & 5.8 & \multirow{3}{*}{.809} \\
\hline & Middle $3^{\text {rd }}$ & 72 & 23.0 & 5 & 6.9 & & 18 & 25.0 & & 3 & 4.2 & \\
\hline & Upper $3^{\text {rd }}$ & 70 & 22.4 & 3 & 4.3 & & 17 & 24.3 & & 3 & 4.3 & \\
\hline \multirow{2}{*}{ Diameter } & $\leq 40 \mathrm{~mm}$ & 160 & 51.1 & 9 & 5.6 & \multirow{2}{*}{.869} & 39 & 24.4 & \multirow{2}{*}{.622} & 10 & 6.2 & \multirow{2}{*}{.497} \\
\hline & $>40 \mathrm{~mm}$ & 153 & 48.9 & 7 & 4.6 & & 42 & 27.4 & & 6 & 3.9 & \\
\hline \multirow{4}{*}{$\begin{array}{l}\text { Depth of } \\
\text { invasion }\end{array}$} & $\mathrm{pT}_{1}$ & 57 & 18.2 & 5 & 8.8 & \multirow{4}{*}{.124} & 13 & 22.8 & \multirow{4}{*}{.258} & 4 & 7.0 & \multirow{4}{*}{.784} \\
\hline & $\mathrm{pT}_{2}$ & 137 & 43.8 & 3 & 2.2 & & 31 & 22.8 & & 7 & 5.1 & \\
\hline & $\mathrm{pT}_{3}$ & 108 & 34.5 & 8 & 8.0 & & 32 & 29.6 & & 5 & 4.6 & \\
\hline & $\mathrm{pT}_{4}$ & 11 & 3.5 & 0 & 0.0 & & 5 & 45.4 & & 0 & 0.0 & \\
\hline \multirow{4}{*}{$\begin{array}{l}\text { Lymph node } \\
\text { status }\end{array}$} & $\mathrm{pN}_{0}$ & 129 & 41.2 & 5 & 3.9 & \multirow{4}{*}{.781} & 33 & 25.6 & \multirow{4}{*}{.252} & 7 & 5.4 & \multirow{4}{*}{.940} \\
\hline & $\mathrm{pN}_{1}$ & 74 & 23.7 & 5 & 6.8 & & 15 & 20.5 & & 3 & 4.1 & \\
\hline & $\mathrm{pN}_{2}$ & 63 & 20.1 & 3 & 4.6 & & 16 & 24.6 & & 3 & 4.6 & \\
\hline & $\mathrm{pN}_{3}$ & 47 & 15.0 & 3 & 6.5 & & 17 & 36.9 & & 3 & 6.5 & \\
\hline \multirow{2}{*}{$\begin{array}{c}\text { Distant } \\
\text { metastases }\end{array}$} & $\mathrm{pM}_{0}$ & 286 & 91.4 & 14 & 4.9 & \multirow{2}{*}{.637} & 67 & 23.4 & \multirow{2}{*}{.003} & 15 & 5.2 & \multirow{2}{*}{.817} \\
\hline & $\mathrm{pM}_{1}$ & 27 & 8.6 & 2 & 7.4 & & 14 & 51.9 & & 1 & 3.7 & \\
\hline \multirow{6}{*}{ Stage } & I a & 52 & 16.6 & 5 & 9.6 & \multirow{6}{*}{.215} & 13 & 25.0 & \multirow{6}{*}{.152} & 4 & 7.7 & \\
\hline & $\mathrm{I} b$ & 67 & 21.4 & 0 & 0.0 & & 15 & 22.4 & & 3 & 4.5 & \\
\hline & II & 54 & 17.3 & 3 & 5.5 & & 10 & 18.5 & & 2 & 3.7 & \\
\hline & III a & 38 & 12.1 & 1 & 2.6 & & 9 & 23.7 & & 2 & 5.3 & (350 \\
\hline & III b & 33 & 10.5 & 2 & 5.7 & & 8 & 22.8 & & 2 & 5.7 & \\
\hline & IV & 69 & 22.1 & 5 & 7.5 & & 26 & 38.8 & & 3 & 4.5 & \\
\hline & G1 & 17 & 5.4 & 2 & 11.8 & & 5 & 29,41 & & 2 & 11.8 & \\
\hline Grading & $\mathrm{G} 2$ & 108 & 34.5 & 7 & 6.5 & .257 & 35 & 32,41 & .126 & 6 & 5.5 & .391 \\
\hline & G3-4 & 188 & 60.1 & 7 & 3.7 & & 41 & 21,80 & & 8 & 4.2 & \\
\hline Microscopic & Intestinal & 184 & 58.8 & 9 & 4.9 & & 47 & 25.5 & & 10 & 5.4 & \\
\hline & Non-intestinal & 129 & 41.2 & 7 & 5.4 & & 34 & 26.3 & & 6 & 4.6 & \\
\hline
\end{tabular}

while limiting the rate of splenic or splenopancreatic resection in order to increase its implementability in western centers is not new [26, 27], and it was confirmed in our experience (adjacent organs were resected only in $16.6 \%$ of cases and a pancreatic resection was carried out only in $3.2 \%$ of cases). The main issue remains how to reach this target without jeopardizing the unique opportunity to perform a $\mathrm{R}_{0}$ resection. The great effort during this implementation study was to spread the main concept of removing the second tier of lymph nodes, or the nodes around the celiac axis and its branches. This was obtained through a strict perioperative protocol and extensive coaching from more experienced surgeons, leading to a satisfactory homogenization of early results. None of the considered outcomes, actually, was significantly influenced by any of the surgeon-related variables. Only a future study on long-term follow-up on the same series will answer the question if this process of standardization offers an equal homogenization in long-term disease control. 
Table 4. Early Results According to Treatment-Related Variables

\begin{tabular}{|c|c|c|c|c|c|c|c|c|c|c|c|c|}
\hline \multirow{4}{*}{$\begin{array}{c}\text { Variable } \\
\begin{array}{c}\text { Gastric } \\
\text { resection }\end{array}\end{array}$} & \multirow{2}{*}{\multicolumn{2}{|c|}{ Pattern No. }} & \multirow{3}{*}{$\begin{array}{c}\% \\
68.1\end{array}$} & \multicolumn{3}{|c|}{ Mortality } & \multicolumn{3}{|c|}{ Morbidity } & \multicolumn{3}{|c|}{ Reoperation } \\
\hline & & & & \multirow{2}{*}{$\begin{array}{c}\text { No. } \\
12\end{array}$} & \multirow{2}{*}{$\begin{array}{c}\% \\
5.6\end{array}$} & $\mathbf{p}$ & No. & $\%$ & $\mathbf{p}$ & No. & $\%$ & $\mathbf{p}$ \\
\hline & Subtotal & 213 & & & & \multirow{2}{*}{.540} & 55 & 25.8 & \multirow{2}{*}{.973} & 12 & 5.6 & \multirow{2}{*}{.540} \\
\hline & Total & 100 & 31.9 & 4 & 4.0 & & 26 & 26.0 & & 4 & 4.0 & \\
\hline \multirow{2}{*}{$\begin{array}{c}\text { Lymph } \\
\text { node } \\
\text { dissection }\end{array}$} & $D_{1 \alpha-\beta}$ & 123 & 51.1 & 12 & 9.7 & \multirow{2}{*}{.002} & 37 & 30.1 & \multirow{2}{*}{.217} & 7 & 5.7 & \multirow{2}{*}{.708} \\
\hline & $\mathrm{D}_{2}$ & 190 & 48.9 & 4 & 2.1 & & 44 & 23.1 & & 9 & 4.7 & \\
\hline \multirow{4}{*}{$\begin{array}{l}\text { Resection } \\
\text { of } \\
\text { adjacent } \\
\text { organs }\end{array}$} & None & 261 & 83.4 & 16 & 6.1 & \multirow{4}{*}{.339} & 65 & 24.9 & \multirow{4}{*}{.091} & 14 & 5.3 & \multirow{4}{*}{.076} \\
\hline & Spleen & 39 & 12.5 & 0 & 0.0 & & 9 & 23.1 & & 0 & 0.0 & \\
\hline & Spleen + pancreas & 10 & 3.2 & 0 & 0.0 & & 6 & 60.0 & & 2 & 20.0 & \\
\hline & Other & 3 & 0.9 & 0 & 0.0 & & 1 & 33.3 & & 0 & 0.0 & \\
\hline \multirow{2}{*}{ Surgeon } & $\# 1$ & 174 & 55.6 & 7 & 4.0 & \multirow{2}{*}{.471} & 37 & 21.3 & \multirow{2}{*}{.039} & 7 & 4.0 & \multirow{2}{*}{.471} \\
\hline & Others & 139 & 44.4 & 9 & 6.5 & & 44 & 31.6 & & 9 & 6.5 & \\
\hline \multirow{8}{*}{$\begin{array}{c}\text { Single } \\
\text { surgeon }\end{array}$} & $\# 1$ & 174 & 55.6 & 7 & 4.0 & \multirow{8}{*}{.344} & 37 & 21.3 & \multirow{8}{*}{.069} & 7 & 4,02 & \\
\hline & $\# 2$ & 34 & 10.9 & 3 & 8.8 & & 13 & 38.2 & & 3 & 8,82 & \\
\hline & $\# 3$ & 29 & 9.3 & 1 & 3.4 & & 6 & 20.7 & & 4 & 13,79 & \\
\hline & $\# 4$ & 18 & 5.7 & 3 & 16.7 & & 9 & 50.0 & & 2 & 11,11 & \\
\hline & $\# 5$ & 13 & 4.1 & 1 & 7.7 & & 2 & 15.4 & & 0 & 0,00 & .105 \\
\hline & $\# 6$ & 11 & 3.5 & 0 & 0.0 & & 3 & 27.3 & & 0 & 0,00 & \\
\hline & $\# 7$ & 10 & 3.2 & 0 & 0.0 & & 2 & 20.0 & & 0 & 0,00 & \\
\hline & Others & 24 & 7.7 & 1 & 4.2 & & 9 & 37.5 & & 0 & 0,00 & \\
\hline & $\mathrm{pR}_{0}$ & 261 & 83.4 & 12 & 4.6 & & 58 & 22.2 & & 14 & 5.4 & \\
\hline Radicality & $\mathrm{pR}_{1}$ & 23 & 7.3 & 1 & 4.3 & .405 & 6 & 26.1 & $<.001$ & 0 & 0.0 & .481 \\
\hline & $\mathrm{pR}_{2}$ & 29 & 9.3 & 3 & 10.3 & & 17 & 58.6 & & 2 & 6.9 & \\
\hline & 1998 & 24 & 7.7 & 2 & 8.3 & & 5 & 20.8 & & 1 & 4.2 & \\
\hline & 1999 & 23 & 7.4 & 1 & 4.3 & & 4 & 17.4 & & 1 & 4.3 & \\
\hline & 2000 & 29 & 9.3 & 3 & 10.3 & & 9 & 31.0 & & 3 & 10.3 & \\
\hline & 2001 & 30 & 9.6 & 0 & 0.0 & & 5 & 16.7 & & 0 & 0.0 & \\
\hline & 2002 & 31 & 9.9 & 1 & 3.2 & & 13 & 41.9 & & 3 & 9.7 & \\
\hline $\begin{array}{c}\text { Accrual } \\
\text { year }\end{array}$ & 2003 & 29 & 9.3 & 2 & 6.9 & .386 & 6 & 20.7 & .234 & 1 & 3.4 & .717 \\
\hline & 2004 & 30 & 9.6 & 0 & 0.0 & & 6 & 20.0 & & 2 & 6.6 & \\
\hline & 2005 & 37 & 11.8 & 3 & 8.1 & & 8 & 21.6 & & 2 & 5.4 & \\
\hline & 2006 & 25 & 7.9 & 3 & 12.0 & & 7 & 28.0 & & 1 & 4.0 & \\
\hline & 2007 & 30 & 9.6 & 0 & 0.0 & & 7 & 23.3 & & 0 & 0.0 & \\
\hline & 2008 & 25 & 7.9 & 1 & 4.0 & & 11 & 44.0 & & 2 & 8.0 & \\
\hline
\end{tabular}


Table 5. Multiple Variable Analysis for Operative Mortality

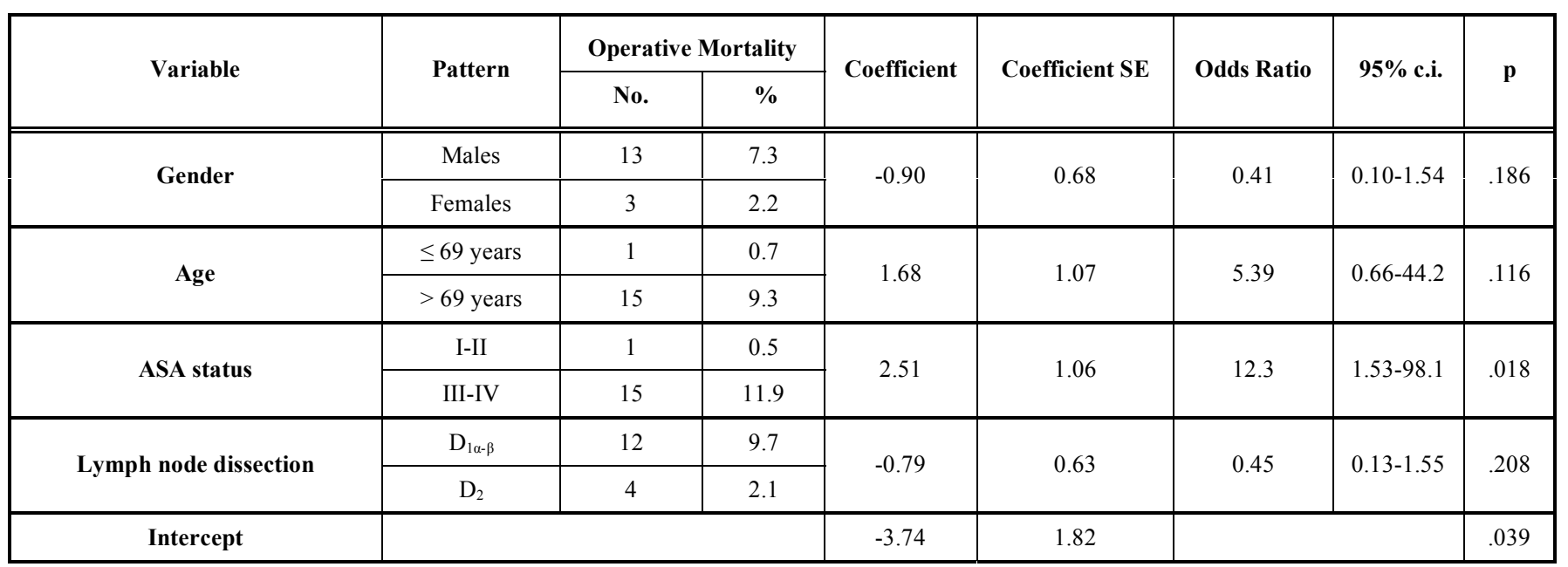

SE: standard error; c.i.: confidence interval; ASA: American Society of Anesthesiologists.

Deviance (likelihood ratio) chi-square $=31.569511 \mathrm{df}=4 \mathrm{P}<.001$.

Hosmer-Lemeshow test $=8.38864 \mathrm{df}=5 \mathrm{P}=.136$.

Table 6. Multiple Variable Analysis for Operative Morbidity

\begin{tabular}{|c|c|c|c|c|c|c|c|c|}
\hline \multirow{2}{*}{ Variable } & \multirow{2}{*}{ Pattern } & \multicolumn{2}{|c|}{ Operative Morbidity } & \multirow{2}{*}{ Coefficient } & \multirow{2}{*}{ Coefficient SE } & \multirow{2}{*}{ Odds Ratio } & \multirow{2}{*}{ 95\% c.i. } & \multirow{2}{*}{$\mathbf{p}$} \\
\hline & & No. & $\%$ & & & & & \\
\hline \multirow{2}{*}{ Age } & $\leq 69$ years & 30 & 19.9 & \multirow{2}{*}{0.19} & \multirow{2}{*}{0.30} & \multirow{2}{*}{1.21} & \multirow{2}{*}{$0.67-2.21$} & \multirow{2}{*}{.519} \\
\hline & $>69$ years & 51 & 31.5 & & & & & \\
\hline \multirow{2}{*}{ ASA status } & I-II & 31 & 16.6 & \multirow{2}{*}{0.98} & \multirow{2}{*}{0.30} & \multirow{2}{*}{2.66} & \multirow{2}{*}{$1.47-4.80$} & \multirow{2}{*}{$<.001$} \\
\hline & III-IV & 50 & 39.7 & & & & & \\
\hline \multirow{2}{*}{ pM } & $\mathrm{pM}_{0}$ & 67 & 23.4 & \multirow{2}{*}{-0.68} & \multirow{2}{*}{1.15} & \multirow{2}{*}{0.50} & \multirow{2}{*}{$0.05-4.87$} & \multirow{2}{*}{.554} \\
\hline & $\mathrm{pM}_{1}$ & 14 & 51.9 & & & & & \\
\hline \multirow{2}{*}{ Surgeon } & $\# 1$ & 37 & 21.3 & \multirow{2}{*}{0.45} & \multirow{2}{*}{0.28} & \multirow{2}{*}{1.58} & \multirow{2}{*}{$0.91-2.76$} & \multirow{2}{*}{.106} \\
\hline & Others & 44 & 31.6 & & & & & \\
\hline \multirow{3}{*}{ pR } & $\mathrm{pR}_{0}$ & 58 & 22.2 & -0.34 & 0.52 & 0.71 & $0.25-1.98$ & .514 \\
\hline & $\mathrm{pR}_{1}$ & 6 & 26.1 & & & & & \\
\hline & $\mathrm{pR}_{2}$ & 17 & 58.6 & 1.81 & 1.16 & 6.13 & $0.62-60.3$ & .119 \\
\hline Intercept & & & & -2.15 & 0.63 & & & $<.001$ \\
\hline
\end{tabular}

SE: standard error; c.i.: confidence interval; ASA: American Society of Anesthesiologists.

Deviance (likelihood ratio) chi-square $=35,666393 \mathrm{df}=5 \mathrm{P}<.001$.

Hosmer-Lemeshow test $=8,109729 \mathrm{df}=5 \mathrm{P}=.15$.

In conclusion, extended lymph node dissection can be safely implemented into the clinical practice of a nondedicated western insitution by limiting the rate of splenopancreatectomy and providing adequate coaching from more expert surgeons.

\section{ACKNOWLEDGEMENTS}

The authors wish to thank Professors Keichi Maruyama, Mitsuru Sasako, Taira Kinoshita and Takeshi Sano of National Cancer Center Hospital, Tokyo, Japan, for sharing and transmitting their skills, passion and unvaluable patience in the treatment of gastric cancer patients.

\section{CONFLICT OF INTEREST}

The authors declare that they have no conflict of interest.

\section{REFERENCES}

[1] Maruyama K. Surgical treatment and end result of gastric cancer. Tokyo: National Cancer Center Press 1986.

[2] Sano T, Sasako M, Yamamoto S, et al. Gastric cancer surgery: morbidity and mortality results from a prospective randomized controlled trial comparing D2 and extended para-aortic lymphadenectomy - Japan Clinical Oncology Group Study 9501. J Clin Oncol 2004; 22: 2767-73.

[3] Siewert JR, Bottcher K, Roder D, et al. Prognostic relevance of systematic lymph node dissection in gastric carcinoma. Br J Surg 1993; 80: 1015-8. 
[4] Siewert JR, Kestlmeier R, Busch R, et al. Benefits of D2 lymph node dissection for patients with gastric cancer and $\mathrm{pN} 0$ and $\mathrm{pN} 1$ lymph node metastases. Br J Surg 1996; 83: 1144-7.

[5] Jaehne J, Meyer H-J, Mascher H, et al. Lymphadenectomy in gastric carcinoma. Arch Surg 1992; 127: 290-4.

[6] Biffi R, Chiappa A, Luca F, et al. Extended lymph-node dissection without routine spleno-pancreatectomy for treatment of gastric cancer: low morbidity and mortality rates in a single center series of 250 patients. J Surg Oncol 2006; 93: 394-400.

[7] De Giuli M, Sasako M, Calgaro M, et al. Morbidity and mortality after D1 and D2 gastrectomy for cancer: interim analysis of the Italian Gastric Cancer Study Group (IGCSG) randomised surgical trial. Eur J Surg Oncol 2004; 30: 303-8.

[8] Marrelli D, Pedrazzani C, Neri A, et al. Complications after extended (D2) and superextended (D3) lymphadenectomy for gastric cancer: analysis of potential risk factors. Ann Surg Oncol 2007;14: 25-33.

[9] Cuschieri A, Weeden S, Fielding J, et al. Patient survival after $\mathrm{D}_{1}$ And $\mathrm{D}_{2}$ resections for gastric cancer: long-term result of the MRC randomised controlled surgical trial. Br J Surg 1999; 79:1522-30.

[10] Bonenkamp JJ, Hermans J, Sasako M, et al, for the Dutch Gastric Cancer Group. Extended lymph node dissection for gastric cancer. N Engl J Med 1999; 340: 908-14.

[11] McCulloch P, Niita EM, Kazi H, et al. Gastrectomy with extended lymphadenectomy for primary treatment of gastric cancer. Br J Surg 2005; 92: 5-13.

[12] Japanese Gastric Cancer Association. Japanese classification of gastric carcinoma - $2^{\text {nd }}$ English Ed. Gastric Cancer 1998; 1: 10-24.

[13] Siewert JR, Feith M, Stein HJ. Biologic and clinical variations of adenocarcinoma at the esophago-gastric junction: relevance of a topographic-anatomic subclassification. J Surg Oncol 2005; 90: 139-46.

[14] Sobin LH, Wittekind $\mathrm{CH}$, International Union Against Cancer (UICC). TNM classification of malignant tumors, $5^{\text {th }}$ ed. New York: Wiley-Liss 1997.

[15] Lauren P. The two histological main types of gastric carcinoma: diffuse and so-called intestinal-type carcinoma. An attempt at a histo-clinical classification. Acta Pathol Microbiol Scand 1965; 64: $31-49$.
[16] Maruyama K, Sasako M, Kinoshita T, et al. Pancreas-preserving total gastrectomy for proximal gastric cancer. World J Surg 1995; 19: 532-6.

[17] Draper NR, Smith H. Applied regression analysis, $3^{\text {rd }}$ ed. New York: Wiley 1998.

[18] Smith JK, McPhee JT, Hill JS, et al. National outcomes after gastric resection for neoplasm. Arch Surg 2007; 142: 387-93.

[19] Msika S, Benhamiche AM, Tazi MA, et al. Improvement of operative mortality after curative resection for gastric cancer: population-based study. World J Surg 2000; 24:1137-42.

[20] Grossmann EM, Longo WE, Virgo KS, et al. Morbidity and mortality of gastrectomy for cancer in department of veterans affairs medical centers. Surgery 2002; 131: 484-90.

[21] Callahan MA, Christos PJ, Gold HT, et al. Influence of surgical subspecialty training on in-hospital mortality for gastrectomy and colectomy patients. Ann Surg 2003; 238: 629-39.

[22] Lee JH, Paik YH, Lee JS, et al. Abdominal shape of gastric cancer patients influences short-term surgical outcomes. Ann Surg Oncol 2007; 14: 1288-94

[23] Mullen JT, Davenport DL, Hutter MM, et al. Impact of body mass index on perioperative outcomes in patients undergoing major intra-abdominal cancer surgery. Ann Surg Oncol 2008; 15: 216472.

[24] Kodera Y, Sasako M, Yamamoto S, et al.; on behalf of the Gastric Cancer Surgery Study Group of Japan Clinical Oncology Group. Identification of risk factors for the development of complications following extended and superextended lymphadenectomies for gastric cancer. Br J Surg 2005; 92: 1103-9.

[25] Tsujinaka T, Sasako M, Yamamoto $\mathrm{S}$, et al. Influence of overweight on surgical complications for gastric cancer: result from randomized control trial comparing D2 and extended para-aortic D3 lymphadenectomy (JCOG9501). Ann Surg Oncol 2007; 14: 355-61.

[26] Elias D. Reflections and proposals for the worldwide standardization of lymphadenectomy for gastric carcinoma. J Surg Oncol 1999; 71:120-22.

[27] Sano T. Tailoring treatments for curable gastric cancer. Br J Surg 2007; 94: 263-4. 